\title{
Rings Whose Annihilating-Ideal Graphs Have Positive Genus*
}

\author{
F. Aliniaeifard ${ }^{\mathrm{a}}$ and M. Behboodi ${ }^{\mathrm{b} \dagger}$ \\ ${ }^{\mathrm{a}, \mathrm{b}}$ Department of Mathematical Sciences, Isfahan University of Technology \\ Isfahan, Iran, 84156-83111 \\ ${ }^{\mathrm{b}}$ School of Mathematics, Institute for Research in Fundamental Sciences (IPM), \\ Tehran Iran, 19395-5746

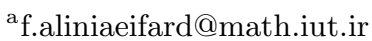 \\ bmbehbood@cc.iut.ac.ir
}

\begin{abstract}
Let $R$ be a commutative ring and $\mathbb{A}(R)$ be the set of ideals with non-zero annihilators. The annihilating-ideal graph of $R$ is defined as the graph $\mathbb{A} \mathbb{G}(R)$ with the vertex set $\mathbb{A}(R)^{*}=\mathbb{A} \backslash\{(0)\}$ and two distinct vertices $I$ and $J$ are adjacent if and only if $I J=(0)$. We investigate commutative rings $R$ whose annihilatingideal graphs have positive genus $\gamma(\mathbb{A} \mathbb{G}(R))$. It is shown that if $R$ is an Artinian ring such that $\gamma(\mathbb{A} \mathbb{G}(R))<\infty$, then $R$ has finitely many ideals or $(R, \mathfrak{m})$ is a Gorenstein ring with maximal ideal $\mathfrak{m}$ and $\mathrm{v} \cdot \operatorname{dim}_{R / \mathfrak{m}} \mathfrak{m} / \mathfrak{m}^{2}=2$. Also, for any two integers $g \geq 0$ and $q>0$, there are finitely many isomorphism classes of Artinian rings $R$ satisfying the conditions: (i) $\gamma(\mathbb{A} G(R))=g$ and (ii) $|R / \mathfrak{m}| \leq q$ for every maximal ideal $\mathfrak{m}$ of $R$. Also, it is shown that if $R$ is a non-domain Noetherian local ring such that $\gamma(\mathbb{A} G(R))<\infty$, then either $R$ is a Gorenstein ring or $R$ is an Artinian ring with finitely many ideals.
\end{abstract}

Key Words: Commutative ring; annihilating-ideal graph; genus of a graph.

2010 Mathematics Subject Classification: 05C10; 13E05; 13E10; 13M05; $16 \mathrm{P} 60$.

\section{Introduction}

The study of algebraic structures, using the properties of graphs, became an exciting research topic in the last twenty years, leading to many fascinating results and

${ }^{*}$ The research of the second author was in part supported by a grant from IPM (No. 89160031)

${ }^{\dagger}$ Corresponding author 
questions. There are many papers on assigning a graph to a ring, for instance see, [3], 4], 5], 6] and [7]. Throughout this paper, $R$ denotes a commutative ring with $1 \neq 0$. Let $X$ be either an element or a subset of $R$. The annihilator of $X$ is the ideal $\operatorname{Ann}(X)=\{a \in R: a X=0\}$. We note that an element $a \in R$ is called a zero-divisor in $R$ if $\operatorname{Ann}(a) \neq\{0\}$ (i.e., $a b=0$ for some nonzero element $b \in R$ ). For any subset $Y$ of $R$, we denote $|Y|$ for the cardinality of $Y$ and let $Y^{*}=Y \backslash\{0\}$. Also, we denote the finite field of order $q$ by $\mathbb{F}_{q}$. Let $V$ be a vector space over the field $\mathbb{F}$. We use the notation $\operatorname{v} \cdot \operatorname{dim}_{\mathbb{F}}(V)$ to denote the dimension of $V$ over the field $\mathbb{F}$.

Let $S_{k}$ denote the sphere with $k$ handles, where $k$ is a non-negative integer, that is, $S_{k}$ is an oriented surface of genus $k$. The genus of a graph $G$, denoted $\gamma(G)$, is the minimal integer $n$ such that the graph can be embedded in $S_{n}$. For details on the notion of embedding a graph in a surface, see, e.g., [16, Chapter 6]. Intuitively, $G$ is embedded in a surface if it can be drawn in the surface so that its edges intersect only at their common vertices. A genus 0 graph is called a planar graph and a genus 1 graph is called a toroidal graph. An infinite graph $G$ has infinite genus $(\gamma(G)=\infty)$, if, for every natural number $n$, there exists a finite subgraph $G_{n}$ of $G$ such that $\gamma\left(G_{n}\right)=n$. We note here that if $H$ is a subgraph of a graph $G$, then $\gamma(H) \leq \gamma(G)$. Let $K_{n}$ denote the complete graph on $n$ vertices; that is, $K_{n}$ has vertex set $V$ with $|V|=n$ and $a-b$ is an edge for every distinct $a, b \in V$. Let $K_{m, n}$ denote the complete bipartite graph; that is, $K_{m, n}$ has vertex set $V$ consisting of the disjoint union of two subsets, $V_{1}$ and $V_{2}$, such that $\left|V_{1}\right|=m$ and $\left|V_{2}\right|=n$, and $a--b$ is an edge if and only if $a \in V_{1}$ and $b \in V_{2}$. We may sometimes write $K_{\left|V_{1}\right|,\left|V_{2}\right|}$ to denote the complete bipartite graph with vertex sets $V_{1}$ and $V_{2}$. Note that $K_{m, n}=K_{n, m}$. It is well known that

$$
\begin{gathered}
\gamma\left(K_{n}\right)=\left\lceil\frac{(n-3)(n-4)}{12}\right\rceil \text { for all } n \geq 3 . \\
\gamma\left(K_{m, n}\right)=\left\lceil\frac{(n-2)(m-2)}{4}\right\rceil \text { for all } n \geq 2 \text { and } m \geq 2 .
\end{gathered}
$$

(see [12]; 10], respectively). For a graph $G$, the degree of a vertex $v$ of $G$ is the number of edges of $G$ incident with $v$.

Let $R$ be a ring. We call an ideal $I$ of $R$, an annihilating-ideal if there exists a non-zero ideal $J$ of $R$ such that $I J=(0)$. We denote $Z(R)$ the sets of zero-divisors, $\mathbb{A}(R)$ for the set of all annihilating-ideals of $R, J(R)$ for the Jacobson radical of $R$, and for an ideal $J$ of $R$, we denote $\mathbb{I}(J)$ for the set $\{I: I$ be an ideal of $R$ and $I \subseteq J\}$. Also, by the annihilating-ideal graph $\mathbb{A} \mathbb{G}(R)$ of $R$ we mean the graph with vertices $\mathbb{A} \mathbb{G}(R)^{*}=\mathbb{A}(R) \backslash\{(0)\}$ such that there is an (undirect) edge between vertices $I$ and $J$ if and only if $I \neq J$ and $I J=(0)$. Thus $\mathbb{A} \mathbb{G}(R)$ is an empty graph if and only if $R$ is an integral domain. Recently, the idea of annihilating-ideal graph of commutative rings 
were introduced by Behboodi and Rakeei in [6, 7] and by Behboodi et al., in [1. The zero-divisor graph of $R, \Gamma(R)$, is an undirect graph with the vertex set $Z(R) \backslash\{0\}$ and two distinct vertices $x, y$ are adjacent if and only if $x y=(0)$. There are some papers which investigate genus of a zero-divisor graph, for instance see [14, [15] and [13]. In particular in [15, Theorem 2], it was shown that for any positive integer $g$, there are finitely many finite commutative rings whose zero-divisor graph has genus $g$.

In the present paper we investigate rings whose annihilating-ideal graphs have positive genus. In Theorem 2.7, it is shown that if $R$ is an Artinian ring and $\gamma(\mathbb{A} \mathbb{G}(R))<\infty$, then $R$ has finitely many ideals or $(R, \mathfrak{m})$ is Gornestein ring with $\operatorname{dim}_{R / \mathfrak{m}} \mathfrak{m} / \mathfrak{m}^{2}=2$ (a Noetherian local ring $(R, \mathfrak{m})$ is called Gorenstein if $\left.\mathrm{v} \cdot \operatorname{dim}_{R / \mathfrak{m}}(\operatorname{Ann}(\mathfrak{m}))=1\right)$. Also, it is shown that, for two integers $g \geq 0$ and $q>0$, there are finitely many Artinian rings $R$ such that (i) $\gamma(\mathbb{A G}(R))=g$ and (ii) $|R / \mathfrak{m}| \leq q$ for every maximal ideal $\mathfrak{m}$ of $R$ (see Theorem 2.10 and Corollary 2.11). Also, the genus of annihilating-ideal graphs of Noetherian rings are studied. It is shown that if $R$ is a Noetherian local ring and $\gamma(\mathbb{A} \mathbb{G}(R))<\infty$, then either $R$ is a domain or all non-trivial ideals of $R$ are vertices of $\mathbb{A} \mathbb{G}(R)$ (see Proposition 3.4). Also if $R$ is a Noetherian ring such that all non-trivial ideals of $R$ are vertices of $\mathbb{A} \mathbb{G}(R)$ and $\gamma(\mathbb{A G}(R))<\infty$, then either $R$ is a Gorenstein ring or $R$ is an Artinian ring with finitely many ideals (see Theorem 3.5). Finally, it is shown that if $R$ is a non-domain Noetherian local ring such that $\gamma(\mathbb{A} \mathbb{G}(R))<\infty$, then either $R$ is a Gorenstein ring or $R$ is an Artinian ring with finitely many ideals (see Corollary [3.6).

\section{The Genus of the Annihilating-Ideal Graphs of Artinian Rings}

The following useful lemma and remark will be used frequently in this paper.

Lemma 2.1 [6, Proposition 1.3] Let $R$ be an Artinian ring. Then every nonzero proper ideal $I$ is a vertex of $\mathbb{A} \mathbb{G}(R)$.

Remark 2.2 It is well known that if $V$ is a vector space over an infinite field $\mathbb{F}$, then $V$ can not be the union of finitely many proper subspaces; see for example [8, p.283].

A local Artinian principal ring is called a special principal ring and has an extremely simple ideal structure: there are only finitely many ideals, each of which is a power of the maximal ideal. 
Lemma 2.3 Let $(R, \mathfrak{m})$ be a local ring with $\mathfrak{m}^{t}=(0)$. If for a positive integer $n$, $\mathrm{v} \cdot \operatorname{dim}_{R / \mathfrak{m}}\left(\mathfrak{m}^{n} / \mathfrak{m}^{n+1}\right)=1$ and $\mathfrak{m}^{n}$ is a finitely generated $R$-module, Then $\mathbb{I}\left(\mathfrak{m}^{n}\right)=$ $\left\{\mathfrak{m}^{i}: t \leq i \leq n\right\}$. Moreover If $n=1$, then $R$ is a special principal ring.

Proof. Since v.dim $R / \mathfrak{m}\left(\mathfrak{m}^{n} / \mathfrak{m}^{n+1}\right)=1$, by using Nakayama's lemma, $\mathfrak{m}^{n}=R x$ for some $x \in R$. Now, let $I$ be a nonzero ideal of $R$ such that $I \subseteq \mathfrak{m}^{n}$. Since $\mathfrak{m}^{t}=(0)$, there exists a natural number $i \geq n$ such that $I \subseteq \mathfrak{m}^{i}$ and $I \nsubseteq \mathfrak{m}^{i+1}$. Let $a \in I \backslash \mathfrak{m}^{i+1}$. We have $a=b x^{i}$, for some $b \in R$. If $b \in m$, then $a \in \mathfrak{m}^{i+1}$, a contradiction. Thus $b$ is unit. Hence $x^{i} \in I$. This implies that $I=\left(x^{i}\right)$, as desired. Thus $\mathbb{I}\left(\mathfrak{m}^{n}\right)=\left\{\mathfrak{m}^{i}: t \leq i \leq n\right\}$. Clearly, if $n=1$, then $R$ is a special principal ring.

Lemma 2.4 Let $(R, \mathfrak{m})$ be an Artinian local ring with $|R / \mathfrak{m}|=\infty, \mathfrak{m}^{3}=(0)$ and $\operatorname{vedim}_{R / \mathfrak{m}} \operatorname{Ann}(\mathfrak{m})=1$. If $\gamma(\mathbb{A} \mathbb{G}(R))<\infty$, then $\operatorname{vedim} R / \mathfrak{m} \mathfrak{m} / \mathfrak{m}^{2} \leq 2$

Proof. First we assume that $\mathrm{v} \cdot \operatorname{dim} R / \mathfrak{m}\left(\mathfrak{m} / \mathfrak{m}^{2}\right)=3$. Then there exist infinitely many subspaces with dimension one. Let $R x / \mathfrak{m}^{2}$ and $R y / \mathfrak{m}^{2}$ be two distinct subspaces of dimension one of $\mathfrak{m} / \mathfrak{m}^{2}$. Since $R x \cong R / \operatorname{Ann}(x)$ and $R x$ has only one nonzero proper $R$-submodule, $\mathfrak{m} / \operatorname{Ann}(x)$ is the only nonzero proper ideal of $R / \operatorname{Ann}(x)$. Therefore, $\mathrm{v} \cdot \operatorname{dim}_{R / \mathfrak{m}} \operatorname{Ann}(x)=2$. Similarly, $\mathbf{v} \cdot \operatorname{dim}_{R / \mathfrak{m}} \operatorname{Ann}(y)=2$. Therefore, $|\mathbb{I}(\operatorname{Ann}(x))|=$ $|\mathbb{I}(\operatorname{Ann}(y))|=\infty$. If $\operatorname{Ann}(x)=\operatorname{Ann}(y)$, then since $(R x) \operatorname{Ann}(x)=(0),(R y) \operatorname{Ann}(x)=$ (0) and $\mathfrak{m}^{2} \operatorname{Ann}(x)=(0)$, we conclude that $K_{|\mathbb{I}(\operatorname{Ann}(x))|, 3}$ is a subgraph of $\mathbb{A} \mathbb{G}(R)$. Thus by the formula for genus of complete bipartite graph, $\gamma(\mathbb{A} \mathbb{G}(R))=\infty$, a contradiction. Thus we may assume that $\operatorname{Ann}(x) \neq \operatorname{Ann}(y)$. If $\operatorname{Ann}(x) \cap \operatorname{Ann}(y)=(0)$, then $(\operatorname{Ann}(x))(\operatorname{Ann}(y))=(0)$ and so $K_{|\mathbb{I}(\operatorname{Ann}(x))|}$ is a subgraph of $\mathbb{A} \mathbb{G}(R)$, and hence, by the formula for complete graphs $\gamma(\mathbb{A} G(R))=\infty$, a contradiction. Therefore, $\operatorname{Ann}(x) \cap \operatorname{Ann}(y) \neq(0)$ and so v.dim $R / \mathfrak{m}(\operatorname{Ann}(x) \cap \operatorname{Ann}(y)) / \mathfrak{m}^{2}=1$.

Suppose that $(R x)(R y) \neq(0)$. Since v.dim $R / \mathfrak{m}(\operatorname{Ann}(x) \cap \operatorname{Ann}(y)) / \mathfrak{m}^{2}=1$, there exists ideal $K$ such that $R x-K-R y$. Let $I_{1}$ be an ideal such that $I_{1} \in \mathbb{I}(\operatorname{Ann}(x)) \backslash$ $\{K, R y\}$ and $v \cdot \operatorname{dim}_{R / \mathfrak{m}} a_{1} / \mathfrak{m}^{2}=1$. Let $J_{1}$ be an ideal such that $J_{1} \in \mathbb{I}(\operatorname{Ann}(x)) \backslash$ $\left\{R x, R y, K, I_{1}, \operatorname{Ann}(x) \cap \operatorname{Ann}(y)\right\}$ such that v.dim $R / \mathfrak{m} J_{1} / \mathfrak{m}^{2}=1$. Let $K_{1}=\operatorname{Ann}\left(I_{1}\right) \cap$ $\operatorname{Ann}\left(J_{1}\right)$. Therefore, $R x-I_{1}-K_{1}-J_{1}-R y$ is a path between $R x$ and $R y$. Now, let $I_{n} \in \mathbb{I}(\operatorname{Ann}(x)) \backslash\left\{I_{i}, J_{i}, K_{i}, R x, R y, \operatorname{Ann}(x) \cap \operatorname{Ann}(y), \operatorname{Ann}(x) \cap \operatorname{Ann}\left(K_{i}\right), i=1,2, . . n-1\right\}$ such that $\mathrm{v} \cdot \operatorname{dim}_{R / \mathfrak{m}} I_{n} / \mathfrak{m}^{2}=1$ and $J_{n} \in \mathbb{I}(\operatorname{Ann}(x)) \backslash\left\{I_{i}, J_{i-1}, K_{i-1}, I_{n}, R x, R y, \operatorname{Ann}(x) \cap\right.$ $\left.\operatorname{Ann}(y), \operatorname{Ann}(x) \cap \operatorname{Ann}\left(K_{i-1}\right), i=1,2, . . n\right\}$ such that $\operatorname{v} \cdot \operatorname{dim}_{R / \mathfrak{m}} J_{n} / \mathfrak{m}^{2}=1$. Suppose that $K_{n}=\operatorname{Ann}\left(x_{n}\right) \cap \operatorname{Ann}\left(y_{n}\right)$, then $R x-I_{n}-K_{n}-J_{n}-R y$ is a path between $R x$ and $R y$. Therefore, there exists infinitely many path between $R x$ and $R y$. Thus either $(R x)(R y)=(0)$ or there exist infinitely many path between $R x$ and $R y$.

Since $R x / \mathfrak{m}^{2}$ and $R y / \mathfrak{m}^{2}$ are two arbitrary distinct one dimensional $R / \mathfrak{m}$-subspaces of 
$\operatorname{Ann}(x)$ and there are infinitely many subspaces of dimension one of $\operatorname{Ann}(x)$, one can easily see that $\gamma(\mathbb{A} \mathbb{G}(R))=\infty$, a contradiction.

Now, we assume that $\operatorname{v} \cdot \operatorname{dim}_{R / \mathfrak{m}}\left(\mathfrak{m} / \mathfrak{m}^{2}\right)>3$. Suppose that $\left\{x_{1}+\mathfrak{m}^{2}, x_{2}+\mathfrak{m}^{2}, x_{3}+\right.$ $\left.\mathfrak{m}^{2}, \ldots, x_{n}+\mathfrak{m}^{2}\right\}$ is a basis for $\mathfrak{m} / \mathfrak{m}^{2}$ over $R / \mathfrak{m}$. Since $R x_{1} \cong R / \operatorname{Ann}\left(x_{1}\right)$ and $R x_{1}$ has one $R$-submodule, v.dim $R / \mathfrak{m}\left(\operatorname{Ann}\left(x_{1}\right) / \mathfrak{m}^{2}\right)=n-1$. Similarly v.dim $R / \mathfrak{m}\left(\operatorname{Ann}\left(x_{2}\right) / \mathfrak{m}^{2}\right)=$ $n-1$. Therefore, $\left.\operatorname{v} \cdot \operatorname{dim}_{R / \mathfrak{m}}\left(\operatorname{Ann}\left(x_{2}\right) \cap \operatorname{Ann}\left(x_{1}\right)\right) / \mathfrak{m}^{2}\right)=n-2$. Since $\left(R x_{1}\right)\left(\operatorname{Ann}\left(x_{2}\right) \cap\right.$ $\left.\operatorname{Ann}\left(x_{1}\right)\right)=(0),\left(R x_{2}\right)\left(\operatorname{Ann}\left(x_{2}\right) \cap \operatorname{Ann}\left(x_{1}\right)\right)=(0)$ and $\mathfrak{m}^{2}\left(\operatorname{Ann}\left(x_{2}\right) \cap \operatorname{Ann}\left(x_{1}\right)\right)=(0)$, we conclude that $K_{\left|\mathbb{I}\left(\left(\operatorname{Ann}\left(x_{2}\right) \cap \operatorname{Ann}\left(x_{1}\right)\right)\right)\right|, 3}$ is a subgraph of $\gamma(\mathbb{A G}(R))$. Thus by the formula for genus of complete bipartite graphs $\gamma(\mathbb{A} \mathbb{G}(R))=\infty$, a contradiction.

Theorem 2.5 Let $(R, \mathfrak{m})$ be an Artinian local ring. If $\gamma(\mathbb{A} \mathbb{G}(R))<\infty$, then $R$ has finitely many ideals or $R$ is a Gorenstein ring with $\operatorname{dim}_{R / \mathfrak{m}} \mathfrak{m} / \mathfrak{m}^{2}=2$.

Proof. If $R$ is a field, then $R$ has finitely many ideals. Thus we can assume that $R$ is not a field. If $|R / \mathfrak{m}|<\infty$, then one can easily see that $R$ is a finite ring and so $R$ has finitely many ideals. Thus we can assume that $|R / \mathfrak{m}|=\infty$ and $\gamma(\mathbb{A} \mathbb{G}(R))=g$ for an integer $g \geq 0$. The proof now proceeds by cases:

Case 1: $\operatorname{vedim}_{R / \mathfrak{m}}(\operatorname{Ann}(\mathfrak{m})) \geq 2$. Then $|\mathbb{I}(\operatorname{Ann}(\mathfrak{m}))|=|\mathbb{I}(\mathfrak{m})|=\infty$ and since $\mathfrak{m A n n}(\mathfrak{m})=$ $(0), K_{|\mathbb{I}(\mathfrak{m})|-4,3}$ is a subgraph of $\mathbb{A} \mathbb{G}(R)$. Hence, by the formula for genus of complete bipartite graphs $\lceil(|\mathbb{I}(\mathfrak{m})|-6) / 4\rceil \leq g$ and so $|\mathbb{I}(\mathfrak{m})| \leq 4 g+6$, a contradiction.

Case 2: $\operatorname{v} \cdot \operatorname{dim}_{R / \mathfrak{m}}(\operatorname{Ann}(\mathfrak{m}))=1$. Since $R$ is an Artinian ring, there exists positive integer $t$ such that $\mathfrak{m}^{t+1}=(0)$ and $\mathfrak{m}^{t} \neq(0)$.

Subcase 1: $t=1$, i.e., $\mathfrak{m}^{2}=(0)$. Then $K_{|\mathbb{I}(\mathfrak{m})|-1}$ is a subgraph of $\mathbb{A} \mathbb{G}(R)$ and so by the formula for genus of complete graphs, $\lceil(|\mathbb{I}(\mathfrak{m})|-6) / 12\rceil \leq g$. Hence $|\mathbb{I}(\mathfrak{m})| \leq 12 g+6$, .i.e., $R$ has at most $12 g+7$ ideals.

Subcase 2: $t=2$, i.e., $\mathfrak{m}^{2} \neq(0)$ and $\mathfrak{m}^{3}=(0)$. Then Lemma 2.4 implies that $\operatorname{v} \cdot \operatorname{dim}_{R / \mathfrak{m}}\left(\mathfrak{m} / \mathfrak{m}^{2}\right) \leq 2$. If $\mathrm{v} \cdot \operatorname{dim}_{R / \mathfrak{m}}\left(\mathfrak{m} / \mathfrak{m}^{2}\right)=1$, then Lemma 2.3 implies that $|\mathbb{I}(\mathfrak{m})|=3$, .i.e., $R$ has four ideals.

Subcase 3: $t \geq 3$. Since $\mathfrak{m}^{3} \mathfrak{m}^{t}=\mathfrak{m}^{3} \mathfrak{m}^{t-1}=\mathfrak{m}^{3} \mathfrak{m}^{t-2}=(0), K_{\left|\mathbb{I}\left(\mathfrak{m}^{3}\right)\right|, 3}$ is a subgraph of $\mathbb{A} \mathbb{G}(R)$. So by the formula for genus of complete bipartite graphs, $\left\lceil\left(\left|\mathbb{I}\left(\mathfrak{m}^{3}\right)\right|-6\right) / 12\right\rceil \leq g$. Hence, $\left|\mathbb{I}\left(\mathfrak{m}^{3}\right)\right| \leq 12 g+6$. If $\operatorname{v} \cdot \operatorname{dim}_{R / \mathfrak{m}}\left(\mathfrak{m}^{t-1} / \mathfrak{m}^{t}\right) \geq 2$, then Remark 2.2 implies that $\left|\mathbb{I}\left(\mathfrak{m}^{t-1}\right)\right|=\infty$. Since $\mathfrak{m}^{t-1} \mathfrak{m}^{t-1}=(0)$ and $t \geq 3, K_{\left|\mathbb{I}\left(\mathfrak{m}^{t-1}\right)\right|-1}$ is a subgraph of $\mathbb{A} \mathbb{G}(R)$. Therefore, $\gamma(\mathbb{A} \mathbb{G}(R))=\infty$, a contradiction. Thus $\mathrm{v} \cdot \operatorname{dim}_{R / \mathfrak{m}}\left(\mathfrak{m}^{t-1} / \mathfrak{m}^{t}\right)=1$. Hence, by Lemma 2.3, there exists $x \in \mathfrak{m}^{t-1}$ such that $\mathfrak{m}^{t-1}=R x$.

Suppose that $\mathrm{v} \cdot \operatorname{dim}_{R / \mathfrak{m}} \mathfrak{m} / \mathfrak{m}^{2}=n \geq 3$. Since $R x \cong R / \operatorname{Ann}(x)$ and $R x$ has only one nonzero proper $R$-submodule, $\mathfrak{m} / \operatorname{Ann}(x)$ is the only nonzero proper ideal of $R / \operatorname{Ann}(x)$. If $\mathfrak{m}^{2} \nsubseteq \operatorname{Ann}(y)$, then $\operatorname{Ann}(x)+\mathfrak{m}^{2}=\mathfrak{m}$, and by Nakayama's lemma, $\operatorname{Ann}(x)=\mathfrak{m}$, a contradiction. Thus $\mathfrak{m}^{2} \subseteq \operatorname{Ann}(x)$ and since $\mathfrak{m} / \operatorname{Ann}(x)$ is the only nonzero proper ideal 
of $R / \operatorname{Ann}(x), \operatorname{v} \cdot \operatorname{dim} R / \mathfrak{m} \operatorname{Ann}(x) / \mathfrak{m}^{2}=n-1$. Let $\left\{y_{1}+\mathfrak{m}^{2}, y_{2}+\mathfrak{m}^{2}, \ldots, y_{n-1}+\mathfrak{m}^{2}, y+\mathfrak{m}^{2}\right\}$ be a basis of $\mathfrak{m} / \mathfrak{m}^{2}$ such that $\left\{y_{1}+\mathfrak{m}^{2}, y_{2}+\mathfrak{m}^{2}, \ldots, y_{n-1}+\mathfrak{m}^{2}\right\}$ be a basis of $\operatorname{Ann}(x) / \mathfrak{m}^{2}$. Since $\mathfrak{m}^{t}$ is the only minimal ideal of $R, \mathfrak{m}^{t} \subseteq R y$ and $|\mathbb{I}(R y)| \geq 3$.

Suppose that $|\mathbb{I}(R y)|=3$. Since $R y \cong R / \operatorname{Ann}(y)$ and $R y$ has only one nonzero proper $R$-submodule, $\mathfrak{m} / \operatorname{Ann}(y)$ is the only nonzero proper ideal of $R / \operatorname{Ann}(y)$. If $\mathfrak{m}^{2} \nsubseteq \operatorname{Ann}(y)$, then $\operatorname{Ann}(y)+\mathfrak{m}^{2}=\mathfrak{m}$, and thus by Nakayama's lemma, Ann $(y)=\mathfrak{m}$, a contradiction. Therefore, $\mathfrak{m}^{2} \subseteq \operatorname{Ann}(y)$. So $\mathfrak{m}^{t-1} \subseteq \operatorname{Ann}(y)$. Hence, $\mathfrak{m}^{t-1} \mathfrak{m}=(0)\left(\mathfrak{m}^{t-1}(R y)=(0)\right.$ and $\left.\mathfrak{m}^{t-1}\left(R y_{1}+\ldots+R y_{n-1}\right)=(0)\right)$, a contradiction. Therefore, $|\mathbb{I}(R y)| \geq 4$.

Assume $|\mathbb{I}(R y)|<\infty$. Since $R y \cong R / \operatorname{Ann}(y), \operatorname{vedim}_{R / \mathfrak{m}}\left(\operatorname{Ann}(y)+\mathfrak{m}^{2}\right) / \mathfrak{m}^{2}=n-1$. Thus $|\mathbb{I}(\operatorname{Ann}(y))|=\infty$ and $K_{|\mathbb{I}(\operatorname{Ann}(y))|,|\mathbb{I}(R y)|}$ is a subgraph of $\mathbb{A} \mathbb{G}(R)$. So by the formula for genus of complete bipartite graphs, $\gamma(\mathbb{A} \mathbb{G}(R))=\infty$.

So we now assume that $|\mathbb{I}(R y)|=\infty$. Suppose that $\mathrm{v} \cdot \operatorname{dim}_{R / \mathfrak{m}} \mathfrak{m}^{2} / \mathfrak{m}^{3} \geq 2$. If $|\mathbb{I}(\operatorname{Ann}(y))| \geq$ 4, then $K_{|\mathbb{I}(R y)|, 3}$ is a subgraph of $\mathbb{A} \mathbb{G}(R)$ and so by the formula for genus of complete bipartite graphs, $\gamma(\mathbb{A} \mathbb{G} R)=\infty$, a contradiction. We may assume that $|\mathbb{I}(\operatorname{Ann}(y))|=3$. $\operatorname{Ann}(y) \nsubseteq \mathfrak{m}^{3}$ (since $\mathfrak{m}^{2} y \cong \mathfrak{m}^{2} /\left(\operatorname{Ann}(y) \cap \mathfrak{m}^{2}\right)$, there exist finitely many ideals between $\operatorname{Ann}(y)$ and $\left.\mathfrak{m}^{2}\right)$. Therefore, there exists $z \in \operatorname{Ann}(y) \backslash \mathfrak{m}^{3}$. Since $|\mathbb{I}(\operatorname{Ann}(y))|=3$, $R z=\operatorname{Ann}(y)$. Since $R z \cong R / \operatorname{Ann}(z)$ and $R z$ has only one nonzero proper $R$ submodule, $\mathfrak{m} / \operatorname{Ann}(z)$ is the only nonzero proper ideal of $R / \operatorname{Ann}(z)$. If $\mathfrak{m}^{2} \nsubseteq \operatorname{Ann}(z)$, then $\operatorname{Ann}(z)+\mathfrak{m}^{2}=\mathfrak{m}$, and thus by Nakayama's lemma, $\operatorname{Ann}(z)=\mathfrak{m}$, a contradiction. Therefore, $\mathfrak{m}^{2} \subseteq \operatorname{Ann}(z)=\operatorname{Ann}(\operatorname{Ann}(y))$. Since $\mathfrak{m}^{2} \operatorname{Ann}(y)=(0), \mathfrak{m}^{2} \mathfrak{m}^{t-1}=(0)$ and $\mathfrak{m}^{t} \mathfrak{m}^{2}=(0), K_{\left|\mathbb{I}\left(\mathfrak{m}^{2}\right)\right|, 3}$ is a subgraph of $\mathbb{A} \mathbb{G}(R)$. So by the formula for genus of complete bipartite graphs, $\gamma(\mathbb{A G}(R))=\infty$, a contradiction. Therefore, v.dim $R / \mathfrak{m} \mathfrak{m}^{2} / \mathfrak{m}^{3}=$ 1 and by Lemma 2.3. $\left|\mathbb{I}\left(\mathfrak{m}^{2}\right)\right|<\infty$. Since $m y \cong \mathfrak{m} / \operatorname{Ann}(y)$ and $|\mathbb{I}(\mathfrak{m} y)|<\infty$, $\operatorname{v} \operatorname{dim}_{R / \mathfrak{m}}\left(\operatorname{Ann}(y)+\mathfrak{m}^{2}\right) / \mathfrak{m}^{2}=n-1$. So $|\mathbb{I}(\operatorname{Ann}(y))|=\infty$. Hence, $K_{|\mathbb{I}(\operatorname{Ann}(y))|,|\mathbb{I}(R y)|}$ is a subgraph of $\mathbb{A} \mathbb{G}(R)$ and by the formula for genus of complete bipartite graphs, $\gamma(\mathbb{A} \mathbb{G}(R))=\infty$, a contradiction. Thus v.dim $R / \mathfrak{m} \mathfrak{m} / \mathfrak{m}^{2} \leq 2$.

Also, if $\mathrm{v} \cdot \operatorname{dim}_{R / \mathfrak{m}} \mathfrak{m} / \mathfrak{m}^{2}=1$, then by Lemma 2.3 , $R$ has finitely many ideals.

Lemma 2.6 Let $R=\prod_{i \in I} R_{i}$ be a product of nonzero rings $R_{i}(i \in I)$. If $\gamma(\mathbb{A} \mathbb{G}(R))<$ $\infty$, then $I$ is finite and for each $i \in I, \gamma\left(\mathbb{A} \mathbb{G}\left(R_{i}\right)\right)<\infty$. Consequently, if $R$ is Artinian, then $\gamma(\mathbb{A G}(R))<\infty$ if and only if $\gamma\left(\mathbb{A} G\left(R_{i}\right)\right)<\infty$ for every $i \in I$.

Proof. Suppose, contrary to our claim, that $|I|=\infty$. Let $I_{1}=\left(R_{i_{1}} \times R_{i_{2}} \times 0 \times \ldots \times 0\right)$ and $I_{2}=\left(0 \times 0 \times R_{i_{3}} \times R_{i_{4}} \times \ldots\right)$. Since $I_{1} I_{2}=(0), K_{\left|\mathbb{I}\left(I_{1}\right)\right|,\left|\mathbb{I}\left(I_{2}\right)\right|}$ is a subgraph of $\mathbb{A} \mathbb{G}(R)$. Since $\left|\mathbb{I}\left(I_{1}\right)\right| \geq 4$ and $\left|\mathbb{I}\left(I_{2}\right)\right|=\infty$, by the formula for genus of complete bipartite graphs, $\gamma(\mathbb{A} G(R))=\infty$, a contradiction. Therefore, $|I|<\infty$ and since $\mathbb{A} \mathbb{G}\left(R_{i}\right)$ is a subgraph of $\mathbb{A} \mathbb{G}(R)$ for every $i \in I$ and $\gamma(\mathbb{A} \mathbb{G}(R))<\infty$, we conclude that $\gamma\left(\mathbb{A} G\left(R_{i}\right)\right)<\infty$ for every $i \in I$. 
Now, suppose that $R$ is an Artinian ring. It is well known that $R \cong R_{1} \times \ldots \times R_{n}$ for some positive integer $n$, where every $R_{i}(i=1,2, \ldots, n)$ is an Artinian local ring. If $n=1$, then the proof is immediate. Now, we may assume that $n \geq 2$. If every $R_{i}$ has finitely many ideals, then $R$ has finitely many ideals and therefore $\gamma(\mathbb{A} \mathbb{G}(R))<\infty$. Without loss of generality, we can assume that $R_{1}$ has infinitely many ideals. Let $I_{1}=\left(0 \times R_{2} \times 0 \times \ldots \times 0\right), I_{2}=\left(\operatorname{Ann}\left(\mathfrak{m}_{1}\right) \times 0 \times \ldots \times 0\right)$ and $I_{3}=\left(\operatorname{Ann}\left(\mathfrak{m}_{1}\right) \times R_{2} \times 0 \times \ldots \times 0\right)$. Then for every ideal $J$ of $R_{1}$, we have $I_{i}(J \times 0 \times \ldots \times 0)=(0)$. Therefore, $K_{\left|\mathbb{I}\left(\mathfrak{m}_{1}\right)\right|-1,3}$ is a subgraph of $\mathbb{A} \mathbb{G}(R)$. Since $\left|\mathbb{I}\left(\mathfrak{m}_{1}\right)\right|=\infty$, by the formula for genus of complete bipartite graphs, $\gamma(\mathbb{A G}(R))=\infty$, a contradiction. Therefore, every $R_{i}$ has finitely many ideals and $\gamma(\mathbb{A} G(R))<\infty$.

Theorem 2.7 Let $R$ be an Artinian ring. If $\gamma(\mathbb{A} G(R))<\infty$, then $R$ has finitely many ideals or $(R, \mathfrak{m})$ is a Gorenstein ring with $\mathrm{v} \cdot \operatorname{dim}_{R / \mathfrak{m}} \mathfrak{m} / \mathfrak{m}^{2}=2$.

Proof. Let $R$ be an Artinian ring. It is well known that $R \cong R_{1} \times \ldots \times R_{n}$ for some positive integer $n$, where every $R_{i}(i=1,2, \ldots, n)$ is an Artinian local ring and addition and multiplication in the product are defined component wise. If $R$ is a local ring, then by Theorem 2.5, $R$ has finitely many ideals or $\operatorname{v} \cdot \operatorname{dim} R / \mathfrak{m} \mathfrak{m} / \mathfrak{m}^{2}=2$. If $n \geq 2$, then as in the proof of Lemma 2.6. every $R_{i}$ has finitely many ideals and therefore $R$ has finitely many ideals.

Lemma 2.8 Let $(R, \mathfrak{m})$ be a Gorenstein ring, $k$ be a positive integer and $q$ be a prime power. If $\mathfrak{m}^{2} \neq(0), \mathfrak{m}^{3}=(0),|R / \mathfrak{m}|=q$ and $\mathrm{v} \cdot \operatorname{dim}_{R / \mathfrak{m}}\left(\mathfrak{m} / \mathfrak{m}^{2}\right)=k>6$, then $\mathbb{A} \mathbb{G}(R)$ contains a copy of $K_{k-6,3}$.

Proof. Since v.dim $R / \mathfrak{m}\left(\mathfrak{m} / \mathfrak{m}^{2}\right)=k$ and $|R|=|R / \mathfrak{m}|\left|\mathfrak{m} / \mathfrak{m}^{2}\right|\left|\mathfrak{m}^{2}\right|$, we conclude that $|R|=q^{k+2}$. Let $\left\{x_{1}+\mathfrak{m}^{2}, \ldots, x_{k}+\mathfrak{m}^{2}\right\}$ be a basis for $\mathfrak{m} / \mathfrak{m}^{2}$ over $R / \mathfrak{m}$. We have $R / \operatorname{Ann}\left(x_{1}\right) \cong R x_{1}$ thus $|R|=\left|R x_{1}\right|\left|\operatorname{Ann}\left(x_{1}\right)\right|$. Since $\mathfrak{m}^{2} \subseteq R x_{1},\left|R x_{1}\right|=\left|\mathfrak{m}^{2}\right||q|=q^{2}$ and $|R|=q^{2}\left|\operatorname{Ann}\left(x_{1}\right)\right|$. Therefore, $\left|\operatorname{Ann}\left(x_{1}\right)\right|=q^{k}$ and $\operatorname{vedim} R / \mathfrak{m}\left(\operatorname{Ann}\left(x_{1}\right) / \mathfrak{m}^{2}\right)=$ $k-2$. Similarly v.dim $R / \mathfrak{m}\left(\operatorname{Ann}\left(x_{2}\right) / \mathfrak{m}^{2}\right)=k-2$. Thus v.dim $R / \mathfrak{m}\left(\left(\operatorname{Ann}\left(x_{1}\right) / \mathfrak{m}^{2}\right) \cap\right.$ $\left.\left(\operatorname{Ann}\left(x_{2}\right) / \mathfrak{m}^{2}\right)\right) \geq k-4$. Let $\left\{x_{i_{1}}+\mathfrak{m}^{2}, \ldots, x_{i_{n}}+\mathfrak{m}^{2}\right\}$ be a basis of $\left(\operatorname{Ann}\left(x_{1}\right) / \mathfrak{m}^{2}\right) \cap$ $\left(\operatorname{Ann}\left(x_{2}\right) / \mathfrak{m}^{2}\right)$. Suppose that $V_{1}=\left\{R x_{1}, R x_{2}, \mathfrak{m}^{2}\right\}$ and $V_{2}=\left\{R x_{i_{1}}, \ldots, R_{x_{i_{n}}}\right\}$. Since $K_{\left|V_{1}\right|,\left|V_{2}\right|}$ is a subgraph of $\mathbb{A} \mathbb{G}(R), K_{k-6,3}$ is a subgraph of $\mathbb{A} \mathbb{G}(R)$.

Lemma 2.9 Let $(R, \mathfrak{m})$ be an Artinian local ring with $|R / \mathfrak{m}|=q$ such that $R$ has finitely many ideals. Then $\left|\mathfrak{m}^{i}\right| \leq q^{\mathbb{I}\left(\mathfrak{m}^{i}\right)}$ for all $i$ and $|R| \leq q^{\mathbb{I}(R)}$

Proof. If $R$ is a field, then the proof is straightforward. Thus we can assume that $R$ is not a field. Since $R$ is an Artinian ring, there exists positive integer $t$ such that 
$\mathfrak{m}^{t} \neq(0)$ and $\mathfrak{m}^{t+1}=(0)$. Let $\mathrm{v} \cdot \operatorname{dim}_{R / \mathfrak{m}}\left(\mathfrak{m}^{i} / \mathfrak{m}^{i+1}\right)=k_{i}$ where $1 \leq i \leq t$. Since $\left|\mathfrak{m}^{i}\right|=\left|\mathfrak{m}^{i} / \mathfrak{m}^{i+1}\right|\left|\mathfrak{m}^{i+1} / \mathfrak{m}^{i+2}\right| \ldots\left|\mathfrak{m}^{t}\right|$ and $k_{i}+\ldots+k_{t} \leq\left|\mathbb{I}\left(\mathfrak{m}^{i}\right)\right|,\left|\mathfrak{m}^{i}\right|=q^{k_{i}+\ldots+k_{t}} \leq q^{\mathbb{I}\left(\mathfrak{m}^{i}\right)}$. Also, $|R|=|R / \mathfrak{m}||m|=q^{k_{1}+\ldots+k_{t}+1} \leq q^{\mathbb{I}(R)}$.

We recall that in [15, Theorem 2], it was shown that for every positive integer $g$ there exist finitely many finite rings with $\gamma(\Gamma(R))=g$. But the result is not true when we replace $\gamma(\Gamma(R))$ with $\gamma(\mathbb{A} \mathbb{G}(R))$. In fact, for every finite field $\mathbb{F}$, the ring $R=\mathbb{F} \times \mathbb{F} \times \mathbb{F} \times \mathbb{F}$ has a toroidal annihilating-ideal graph $\left(\right.$ since $\gamma(\mathbb{A} G(R))=\gamma\left(\mathbb{A} G\left(\mathbb{Z}_{2} \times \mathbb{Z}_{2} \times \mathbb{Z}_{2} \times \mathbb{Z}_{2}\right)\right)=$ 1 ). Next, we proceed to show that for every integers $q>0$ and $g \geq 0$, there are finitely many Artinian rings $R$, such that $\gamma(\mathbb{A G}(R))=g$ and $|R / \mathfrak{m}| \leq q$ for every maximal ideal $\mathfrak{m}$ of $R$. We first prove a reduced form of the result.

Theorem 2.10 For a prime power $q$ and an integer $g \geq 0$, there are finitely many Artinian local rings $(R, \mathfrak{m})$ satisfying the following conditions:

(1) $\gamma(\mathbb{A} \mathbb{G}(R))=g$,

(2) $|R / \mathfrak{m}|=q$.

Proof. Suppose that $R$ is an Artinian local ring with $|R / \mathfrak{m}|=q$ and $\gamma(\mathbb{A} \mathbb{G}(R))=g$. Since there exists only one field of order $q$, we can assume that $R$ is not a field. Thus there exists a positive integer $t$ such that $\mathfrak{m}^{t} \neq(0), \mathfrak{m}^{t+1}=(0)$. Since $R$ is an Artinian ring and $|R / \mathfrak{m}|=q, R$ is a finite ring. It is sufficient to show that $|R|$ is bounded by a constant depending only on $g$ and $q$. The proof now proceeds by cases:

Case 1: $\operatorname{vedim}_{R / \mathfrak{m}}(\operatorname{Ann}(\mathfrak{m})) \geq 2$. Since $\operatorname{Ann}(\mathfrak{m}) \mathfrak{m}=(0), K_{|\mathbb{I}(R)|-5,3}$ is a subgraph of $\mathbb{A} \mathbb{G}(R)$. By the formula for the genus of complete bipartite graphs, $\lceil(|\mathbb{I}(R)|-7) / 4\rceil \leq g$ thus $|\mathbb{I}(R)| \leq 4 g+7$ and therefore by Lemma 2.9 , $|R| \leq q^{4 g+7}$.

Case 2: $\operatorname{v} \cdot \operatorname{dim}_{R / \mathfrak{m}}(\operatorname{Ann}(\mathfrak{m}))=1$.

Subcase 1: $t=1$, i.e., $\mathfrak{m}^{2}=0$. Since $|R|=|R / \mathfrak{m}||\mathfrak{m}|$ and $\mathbf{v} \cdot \operatorname{dim}_{R / \mathfrak{m}} \mathfrak{m}=1$, we conclude that $|R|=q^{2}$.

Subcase 2: $t=2$, i.e., $\mathfrak{m}^{2} \neq(0)$ and $\mathfrak{m}^{3}=(0)$. Let $\mathrm{v} \cdot \operatorname{dim}_{R / \mathfrak{m}}\left(\mathfrak{m} / \mathfrak{m}^{2}\right)=k$. Then by Lemma 2.8. $\mathbb{A} \mathbb{G}(R)$ contains a copy of $K_{k-6,3}$. By the formula for the genus of complete bipartite graphs, $\lceil(k-8) / 4\rceil \leq g$ thus $|k| \leq 4 g+8$. Since $|R|=|R / \mathfrak{m}|\left|\mathfrak{m} / \mathfrak{m}^{2}\right|\left|\mathfrak{m}^{2}\right|$ and v.dim $R / \mathfrak{m} \mathfrak{m}^{2}=1$, we conclude that $|R| \leq q^{2+4 g+8}$.

Subcase 3: $t \geq 3$. The proof will be divided into three steps.

Step 1: We prove that $\left|\mathfrak{m}^{3}\right|$ is bounded by a constant depending only on $g$ and $q$. If $t=3,4,5$, then $\left(\mathfrak{m}^{3}\right)^{2}=(0)$. Therefore, $K_{\left|\mathbb{I}\left(\mathfrak{m}^{3}\right)\right|-1}$ is a subgraph of $\mathbb{A} \mathbb{G}(R)$ and by the formula for the genus of complete graphs, $\left\lceil\left(\left|\mathbb{I}\left(\mathfrak{m}^{3}\right)\right|-5\right) / 12\right\rceil \leq g$ thus $\left|\mathbb{I}\left(\mathfrak{m}^{3}\right)\right| \leq 12 g+5$ and by Lemma $2.9,\left|\mathfrak{m}^{3}\right| \leq q^{12 g+5}$.

If $t \geq 5$ then $\mathfrak{m}^{t} \mathfrak{m}^{3}=(0), \mathfrak{m}^{t-1} \mathfrak{m}^{3}=(0), \mathfrak{m}^{t-2} \mathfrak{m}^{3}=(0)$. Therefore, $K_{\left|\mathbb{I}\left(\mathfrak{m}^{3}\right)\right|-4,3}$ is a subgraph of $\mathbb{A} \mathbb{G}(R)$ and by the formula for the genus of complete bipartite graphs, 
$\left\lceil\left(\left|I\left(\mathfrak{m}^{3}\right)\right|-5\right) / 4\right\rceil \leq g$. Thus $\left|\mathbb{I}\left(\mathfrak{m}^{3}\right)\right| \leq 4 g+5$ and by Lemma 2.9 , $\left|\mathfrak{m}^{3}\right| \leq q^{4 g+5}$.

Step 2: We prove that $\left|\mathfrak{m}^{2}\right|$ is bounded by a constant depending only on $g$ and $q$. If $\operatorname{v} \cdot \operatorname{dim}_{R / \mathfrak{m}}\left(\mathfrak{m}^{2} / \mathfrak{m}^{3}\right)=1$, then $\left|\mathfrak{m}^{2}\right|=q\left|\mathfrak{m}^{3}\right|$. Now, by Step 1 , we conclude that $\left|\mathfrak{m}^{2}\right|$ is also bounded by a constant depending only on $g$ and $q$. Suppose that $\mathrm{v} \cdot \operatorname{dim} \operatorname{dim}_{R / \mathfrak{m}}\left(\mathfrak{m}^{2} / \mathfrak{m}^{3}\right)=$ $k \geq 2$. Let $\left\{x_{1}+\mathfrak{m}^{3}, \ldots, x_{k}+\mathfrak{m}^{3}\right\}$ be a basis for $\mathfrak{m}^{2} / \mathfrak{m}^{3}$ over $R / \mathfrak{m}$. Since $\mathfrak{m}^{t} \varsubsetneqq R x_{i}$, $\left|\mathbb{I}\left(R x_{i}\right)\right| \geq 3$, for each $i(1 \leq i \leq k)$. If $\left|\mathbb{I}\left(R x_{1}\right)\right|=\left|\mathbb{I}\left(R x_{2}\right)\right|=3$, then since $R x_{i} \cong R / \operatorname{Ann}\left(x_{i}\right), 1 \leq i \leq 2$, there is no ideals between $\operatorname{Ann}\left(x_{i}\right), 1 \leq i \leq 2$ and $\mathfrak{m}$. Now, if $\mathfrak{m}^{2} \nsubseteq \operatorname{Ann}\left(x_{i}\right), 1 \leq i \leq 2$, then $\operatorname{Ann}\left(x_{i}\right)+\mathfrak{m}^{2}=\mathfrak{m}, 1 \leq i \leq 2$ and by Nakayama's lemma, $\operatorname{Ann}\left(x_{i}\right)=\mathfrak{m}, 1 \leq i \leq 2$, a contradiction. Therefore, $\mathfrak{m}^{2} \subseteq \operatorname{Ann}\left(x_{i}\right), 1 \leq i \leq 2$. Since $\left(\mathfrak{m}^{2}\right)\left(R x_{i}\right)=(0), 1 \leq i \leq 2$ and $\mathfrak{m}^{t} \mathfrak{m}^{2}=(0), K_{\left|\mathbb{I}\left(\mathfrak{m}^{2}\right)\right|-2,3}$ is a subgraph of $\mathbb{A} \mathbb{G}(R)$. By the formula for genus of complete bipartite graphs, $\left\lceil\left(\left|\mathbb{I}\left(\mathfrak{m}^{2}\right)\right|-4\right) / 4\right\rceil \leq g$, thus $\left|\mathbb{I}\left(\mathfrak{m}^{2}\right)\right| \leq 4 g+4$ and by Lemma $2.9,\left|\mathfrak{m}^{2}\right| \leq q^{4 g+4}$. Without loss of generality we can assume that $\left|\mathbb{I}\left(R x_{1}\right)\right| \geq 4$. Then $K_{\left|\mathbb{I}\left(\operatorname{Ann}\left(x_{1}\right)\right)\right|-4,3}$ is a subgraph of $\mathbb{A} \mathbb{G}(R)$. By the formula for genus of complete bipartite graphs $\left\lceil\left(\left|\mathbb{I}\left(\operatorname{Ann}\left(x_{1}\right)\right)\right|-6\right) / 4\right\rceil \leq g$, thus $\left|\mathbb{I}\left(\operatorname{Ann}\left(x_{1}\right)\right)\right| \leq 4 g+6$. Since $\left|\mathbb{I}\left(\operatorname{Ann}\left(x_{1}\right) \cap \mathfrak{m}^{2}\right)\right| \leq \mid \mathbb{I}\left(\operatorname{Ann}\left(x_{1}\right) \mid\right.$ and dimension of every vector space less than or equal to cardinality of each its generating set, we conclude that $\operatorname{v} \cdot \operatorname{dim}_{R / \mathfrak{m}}\left(\left(\operatorname{Ann}\left(x_{1}\right) \cap \mathfrak{m}^{2}\right)+\mathfrak{m}^{3}\right) / \mathfrak{m}^{3} \leq \mid \mathbb{I}\left(\operatorname{Ann}\left(x_{1}\right) \mid\right.$. Let $\theta: \mathfrak{m}^{2} \rightarrow \mathfrak{m}^{2} x_{1}$ be the group homomorphism defined by multiplication by $x_{1}$. Then we have $\left|\mathfrak{m}^{2}\right| /\left|\mathfrak{m}^{2} \cap \operatorname{Ann}\left(x_{1}\right)\right|=$ $\left|\mathfrak{m}^{2} x\right|$. It follows that $\left|\mathfrak{m}^{2}\right| \leq\left|\mathfrak{m}^{3}\right|\left|\mathfrak{m}^{2} \cap \operatorname{Ann}\left(x_{1}\right)\right| \leq\left|\mathfrak{m}^{3}\right|\left|\mathfrak{m}^{2} \cap \operatorname{Ann}\left(x_{1}\right)+\mathfrak{m}^{3}\right| \leq$ $\left|\mathfrak{m}^{3}\right|{ }^{2} q^{\left|\mathbb{I}\left(\operatorname{Ann}\left(x_{1}\right)\right)\right|} \leq\left|\mathfrak{m}^{3}\right|{ }^{2} q^{4 g+6}$. Now, by Step 1, we conclude that $\left|\mathfrak{m}^{2}\right|$ is also bounded by a constant depending only on $g$ and $q$.

Step 3: Now, we show that $|\mathfrak{m}|$ is bounded by a constant depending only on $g$ and q. We can assume that $v \cdot \operatorname{dim}_{R / \mathfrak{m}}\left(\mathfrak{m} / \mathfrak{m}^{2}\right)=k \geq 5$. Let $\left\{x_{1}+\mathfrak{m}^{2}, \ldots, x_{k}+\mathfrak{m}^{2}\right\}$ be a basis for $\mathfrak{m} / \mathfrak{m}^{2}$ over $R / \mathfrak{m}$. Since $\mathfrak{m}^{t} \varsubsetneqq R x_{i},\left|\mathbb{I}\left(R x_{i}\right)\right| \geq 3$, for each $i(1 \leq i \leq k)$. If $\left|\mathbb{I}\left(R x_{1}\right)\right|=\left|\mathbb{I}\left(R x_{2}\right)\right|=3$, then since $R x_{i} \cong R / \operatorname{Ann}\left(x_{i}\right), 1 \leq i \leq 2$, there is no ideals between $\operatorname{Ann}\left(x_{i}\right), 1 \leq i \leq 2$ and $\mathfrak{m}$. If $\mathfrak{m}^{2} \nsubseteq \operatorname{Ann}\left(x_{i}\right)$, for $i=1$ or 2 , then $\operatorname{Ann}\left(x_{i}\right)+\mathfrak{m}^{2}=\mathfrak{m}$, and so by Nakayama's lemma, $\operatorname{Ann}\left(x_{i}\right)=\mathfrak{m}$. Since $\operatorname{v} \cdot \operatorname{dim}_{R / \mathfrak{m}}(\operatorname{Ann}(\mathfrak{m}))=1$ and $\mathfrak{m}^{t} \mathfrak{m}=(0), \operatorname{Ann}(\mathfrak{m})=\mathfrak{m}^{t}$. Thus $R x_{i} \subseteq \mathfrak{m}^{t} \subseteq \mathfrak{m}^{2}$, a contradiction. Therefore, $\mathfrak{m}^{2} \subseteq \operatorname{Ann}\left(x_{1}\right) \cap \operatorname{Ann}\left(x_{2}\right)$. If v.dim $R / \mathfrak{m}\left(\operatorname{Ann}\left(x_{i}\right) / \mathfrak{m}^{2}\right) \leq k-2$ for $i=1$ or 2 , then there exists proper ideal $I \subseteq R$ such that $\operatorname{Ann}\left(x_{i}\right) / \mathfrak{m}^{2} \varsubsetneqq I / \mathfrak{m}^{2} \varsubsetneqq \mathfrak{m} / \mathfrak{m}^{2}$, and hence $\operatorname{Ann}\left(x_{i}\right) \varsubsetneqq I \varsubsetneqq \mathfrak{m}$, a contradiction. Thus v.dim $R / \mathfrak{m}\left(\operatorname{Ann}\left(x_{i}\right) / \mathfrak{m}^{2}\right) \geq k-1$ for $i=1$, 2. It follows that v.dim $R / \mathfrak{m}\left(\operatorname{Ann}\left(x_{1}\right) \cap \operatorname{Ann}\left(x_{2}\right)\right) \geq k-2$. Since $R x_{1}\left(\operatorname{Ann}\left(x_{1}\right) \cap\right.$ $\left.\operatorname{Ann}\left(x_{2}\right)\right)=(0), R x_{2}\left(\operatorname{Ann}\left(x_{1}\right) \cap \operatorname{Ann}\left(x_{2}\right)\right)=(0)$ and $\mathfrak{m}^{t}\left(\operatorname{Ann}\left(x_{1}\right) \cap \operatorname{Ann}\left(x_{2}\right)\right)=(0)$ and $\left|\mathbb{I}\left(\operatorname{Ann}\left(x_{1}\right) \cap \operatorname{Ann}\left(x_{2}\right)\right) \backslash\left\{(0), R x_{1}, R x_{2}\right\}\right| \geq k-5$, we conclude that $K_{k-5,3}$ is a subgraph of $\mathbb{A} \mathbb{G}(R)$. Now, by the formula for genus of complete bipartite graphs, $k \leq 5 g+6$ hence $|\mathfrak{m}| \leq\left|\mathfrak{m}^{2}\right|{ }^{2} q^{5 g+6}$. Thus by Step 2 , we conclude that $|\mathfrak{m}|$ is also bounded by a constant depending only on $g$ and $q$.

Finally, without loss of generality we can assume that $\left|\mathbb{I}\left(R x_{1}\right)\right| \geq 4$. Thus $K_{\left|\mathbb{I}\left(\operatorname{Ann}\left(x_{1}\right)\right)\right|-4,3}$ 
is a subgraph of $\mathbb{A G}(R)$. By the formula for genus of complete bipartite graphs, $\left\lceil\mid\left(\mathbb{I}\left(\operatorname{Ann}\left(x_{1}\right)\right) \mid-6\right) / 4\right\rceil \leq g$ and hence $\left|\mathbb{I}\left(\operatorname{Ann}\left(x_{1}\right)\right)\right| \leq 4 g+6$. Since $\left|\mathbb{I}\left(\operatorname{Ann}\left(x_{1}\right) \cap \mathfrak{m}^{2}\right)\right| \leq$ $\mid \mathbb{I}\left(\operatorname{Ann}\left(x_{1}\right) \mid\right.$ and the dimension of every vector space less than or equal to cardinality of each its generating set, we conclude that $\mathrm{v} \cdot \operatorname{dim}_{R / \mathfrak{m}}\left(\operatorname{Ann}\left(x_{1}\right)+\mathfrak{m}^{2}\right) / \mathfrak{m}^{2} \leq\left|\mathbb{I}\left(\operatorname{Ann}\left(x_{1}\right)\right)\right|$. Let $\vartheta: \mathfrak{m} \rightarrow \mathfrak{m} x_{1}$ be the group homomorphism defined by multiplication by $x_{1}$. Then

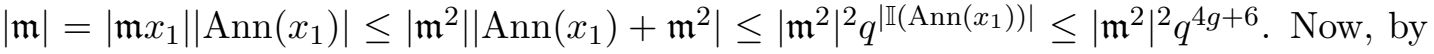
Step 2, we conclude that $|\mathfrak{m}|$ is also bounded by a constant depending only on $g$ and $q$. This finishes the proof, since $|R|=|R / \mathfrak{m}||\mathfrak{m}|$ and so $|R|$ is bounded by a constant depending only on $g$ and $q$.

Corollary 2.11 For integers $q>0$ and $g \geq 0$, there are finitely many Artinian rings $R$ satisfying the following conditions:

(1) $\gamma(\mathbb{A} \mathbb{G}(R))=g$,

(2) $|R / \mathfrak{m}| \leq q$ for any maximal ideal $\mathfrak{m}$ of $R$.

Proof. Let $R$ be an Artinian ring with $\gamma(\mathbb{A G}(R))=g$. It is well known that $R \cong$ $R_{1} \times \ldots \times R_{n}$ for some positive integer $n$, where every $R_{i}(i=1,2, \ldots, n)$, is an Artinian local ring. Since $\left|R_{i} / \mathfrak{m}_{i}\right| \leq q$, every $R_{i}$ is a finite local ring. For ease of exposition, we assume that $\left|R_{i}\right| \geq\left|R_{i+1}\right|$ for $i=1,2, \ldots, n-1$ as there is no loss of generality with this assumption.

It sufficient to show that $|R|$ is bounded by a constant depending only on $g$ and $q$. Furthermore, $\left|R_{1}\right| \geq\left|R_{i}\right|$ for all $i$, it is enough to bound $R_{1}$ by a constant depending only on $g$ and $q$. By a slight abuse of notation, we denote the set $0 \times \ldots \times R_{i} \times \ldots \times 0$ by $R_{i}$, and the set $R_{1} \times \ldots \times R_{i-1} \times 0 \times R_{i+1} \times \ldots R_{n}$ by $\bar{R}_{i}$.

If $n \geq 3$, then $\left|\mathbb{I}\left(\overline{R_{1}^{*}}\right)\right| \geq 3$. Thus $\mathbb{A} \mathbb{G}(R)$ contains a copy of $K_{\left|\mathbb{I}\left(R_{1}\right)\right|-1,3}$. By the formula for the genus of complete bipartite graphs, we have $\left\lceil\left(\left|\mathbb{I}\left(R_{1}\right)\right|-3\right) / 4\right\rceil \leq g$ so $\left|\mathbb{I}\left(R_{1}\right)\right| \leq 4 g+3$ and thus by Lemma 2.9, $\left|R_{1}\right| \leq q^{4 g+3}$.

Suppose that $n=2$. Let $\left|\mathbb{I}\left(R_{2}\right)\right| \geq 4$, this means $\mathbb{A} \mathbb{G}(R)$ contains a copy of $K_{\left|\mathbb{I}\left(R_{1}\right)\right|-1,3}$, which as above gives $\left|R_{1}\right| \leq q^{4 g+3}$.

Let $\left|\mathbb{I}\left(R_{2}\right)\right| \leq 3$. If $R_{1}$ be a field, then $\left|R_{1}\right| \leq q$. Thus we may assume that $R_{1}$ is not a field. Let $\mathfrak{m} \neq(0)$ be the maximal ideal of $R_{1}$. Since $R_{1}$ is an Artinian ring, $\operatorname{Ann}(\mathfrak{m}) \neq(0)$. Assume that $I=(\mathfrak{m}, 0), J_{1}=(\operatorname{Ann}(\mathfrak{m}), 0), J_{2}=\left(\operatorname{Ann}(\mathfrak{m}), R_{2}\right)$ and $J_{3}=\left(0, R_{2}\right)$. Then $I J_{i}=(0)$ for $1 \leq i \leq 3$. Therefore, $\mathbb{A} \mathbb{G}(R)$ contains a copy of $K_{\left|\mathbb{I}\left(R_{1}\right)\right|-2,3}$. Now, by the formula for the genus of complete bipartite graphs, $\left\lceil\left(\left|\mathbb{I}\left(R_{1}\right)\right|-4\right) / 4\right\rceil \leq g$ and so $\left|\mathbb{I}\left(R_{1}\right)\right| \leq 4 g+4$. Therefore, by Lemma 2.9, $\left|R_{1}\right| \leq q^{4 g+4}$. Finally, suppose that $n=1$. Let $R$ be an Artinian local ring with $|R / \mathfrak{m}|=q$. Then by Theorem 2.10, $|R|$ is bounded by a constant depending only on $g$ and $q$. 


\section{The Genus of Annihilating-Ideal Graphs of Noetherian Rings}

In this section, we investigate the genus of annihilating-ideal graphs of Noetherian rings.

Let $R$ be a ring. We say that the annihilating-ideal graph $\mathbb{A} \mathbb{G}(R)$ has assenting chain condition (ACC) (resp., descending chain condition (DCC)) on vertices if $R$ has ACC (resp., DCC) on $\mathbb{A}(R)$.

First, we need the following useful lemmas.

Lemma 3.1 [6, Theorem 1.1] Let $R$ be a ring that is not a domain. Then $\mathbb{A} \mathbb{G}(R)$ has $A C C$ (resp., DCC) on vertices if and only if $R$ is a Noetherian (resp., an Artinian) ring.

Lemma 3.2 [6, Proposition 1.7] Let $R$ be a Noetherian ring. If all non-trivial ideals of $R$ are vertices of $\mathbb{A} \mathbb{G}(R)$, then $R$ has finitely many maximal ideals.

By Lemma 2.1, any non-trivial ideal of an Artinian ring $R$ is a vertex of $\mathbb{A} \mathbb{G}(R)$. Now, a natural question is posed: If $R$ is a Noetherian ring and all non-trivial ideals of $R$ are vertices of $\mathbb{A} \mathbb{G}(R)$, then is $R$ an Artinian ring? the answer of this question is negative (see [1, Example 17]). Next, we have the following result.

Proposition 3.3 Let $(R, \mathfrak{m})$ be a Gorenstein ring. Then all non-trivial ideals of $R$ are vertices of $\mathbb{A} \mathbb{G}(R)$.

Proof. If $(R, \mathfrak{m})$ is a Gorenstein ring, then $v \cdot \operatorname{dim}_{R / \mathfrak{m}}(\operatorname{Ann}(\mathfrak{m}))=1$. It follows that $\operatorname{Ann}(\mathfrak{m}) \neq(0)$ and so $\mathfrak{m}$ is a vertex of $\mathbb{A} \mathbb{G}(R)$, i.e., all non-trivial ideals of $R$ are vertices of $\mathbb{A} \mathbb{G}(R)$.

Proposition 3.4 Let $R$ be a Noetherian local ring. If $\gamma(\mathbb{A G}(R))<\infty$, then either $R$ is a domain or all non-trivial ideals of $R$ are vertices of $\mathbb{A} \mathbb{G}(R)$.

Proof. Let $R$ be a Noetherian ring and $\gamma(\mathbb{A} \mathbb{G}(R))<\infty$. We can assume that $R$ is not a domain. If $R$ is an Artinian ring, then by Lemma 2.1, every ideal of $R$ is a vertex of $\mathbb{A} \mathbb{G}(R)$. Suppose that $R$ is not an Artinian ring. By Lemma 3.1, there exists an infinite descending chain $I_{1} \supsetneqq I_{2} \supsetneqq \ldots \supsetneqq I_{n} \supsetneqq \ldots$ in $\mathbb{A}(R)$. Suppose that $P$ is a maximal element in $\mathbb{A}(R)$ such that $I_{1} \subseteq P$. Clearly $P$ is maximal among all annihilators of non-zero elements of $R$ and so by [9, Theorem 6], $P$ is a prime ideal. Also, $\left\{I_{n} \mid n \in \mathbb{N}\right\} \subseteq P$ and 
hence $\left|\mathbb{I}(P)^{*}\right|=\infty$. Set $I=\operatorname{Ann}(P)$. If $\left|\mathbb{I}(I)^{*}\right| \geq 3$, then $K_{\left|\mathbb{I}(I)^{*}\right|,\left|\mathbb{I}(P)^{*}\right|}$ is a subgraph of $\mathbb{A} \mathbb{G}(R)$. Since $\left|\mathbb{I}(P)^{*}\right|=\infty, \gamma(\mathbb{A} \mathbb{G}(R))=\infty$, a contradiction. If $\left|\mathbb{I}(I)^{*}\right|=2$, then we must have $I=R x$ for some $x \in R$, for otherwise, there are two nonzero distinct ideals $R z, R x \subseteq I$, and so $\left|\mathbb{I}(I)^{*}\right| \geq 3$, a contradiction. Since $\operatorname{Ann}(I)=\operatorname{Ann}(x)=P$, $R x \cong R / \operatorname{Ann}(x)$ and $R x$ has only one nonzero $R$-submodule, we conclude that there is no ideals between $P$ and $\mathfrak{m}$. Since $P \subseteq P+\mathfrak{m}^{2} \subseteq \mathfrak{m}$, either $P+\mathfrak{m}^{2}=P$ or $P+\mathfrak{m}^{2}=\mathfrak{m}$. If $P+\mathfrak{m}^{2}=P$, then $\mathfrak{m}^{2} \subseteq P$. It follows that $P=\mathfrak{m}$. Also, if $P+\mathfrak{m}^{2}=\mathfrak{m}$, then by Nakayama's lemma, $P=\mathfrak{m}$. Thus $I=R x$ is a simple $R$-module, contrary to $\left|\mathbb{I}(I)^{*}\right|=2$. Thus $\left|\mathbb{I}(I)^{*}\right|=1$, i.e., $I$ is a simple $R$-module. This implies that $P=\mathfrak{m}$. Thus $\mathfrak{m}$ is a vertex of $\mathbb{A} \mathbb{G}(R)$, i.e., all non-trivial ideals of $R$ are vertices of $\mathbb{A} \mathbb{G}(R)$.

Theorem 3.5 Let $R$ be a Noetherian ring such that all non-trivial ideals of $R$ are vertices of $\mathbb{A} G(R)$. If $\gamma(\mathbb{A}(R))<\infty$, then either $R$ is a Gorenstein ring or $R$ is an Artinian ring with finitely many ideals.

Proof. Suppose that $R$ is a Noetherian ring, all non-trivial ideals of $R$ are vertices of $\mathbb{A} \mathbb{G}(R)$ and $\gamma(\mathbb{A} \mathbb{G}(R))<\infty$. Suppose that $R$ is a local ring with maximal ideal $\mathfrak{m}$. We can assume that $R$ is not a Gorenstein ring. Therefore $\operatorname{v} \cdot \operatorname{dim}_{R / \mathfrak{m}}(\operatorname{Ann}(\mathfrak{m})) \geq 2$. It follows that $\left|\operatorname{Ann}(\mathfrak{m})^{*}\right| \geq 3$ and $\operatorname{Ann}(\mathfrak{m}) \mathfrak{m}=(0)$. If $|\mathbb{I}(R)|=\infty$, then we conclude that $K_{|\mathbb{I}(R)|-5,3}$ is a subgraph of $\mathbb{A} \mathbb{G}(R)$. Thus by the formula for the genus of complete bipartite graphs, we have $|\mathbb{I}(R)|=\infty$, a contradiction. Thus $|\mathbb{I}(R)|<\infty R$, i.e., $R$ is an Artinian ring with finitely many ideals.

Now, we can assume that $R$ is not a local ring. It is sufficient to shown that every prime ideal is a maximal ideal. By Lemma 3.2, $R$ has finitely many maximal ideals. Suppose that $k \geq 2, \mathfrak{m}_{1}, \ldots, \mathfrak{m}_{k}$ are all maximal ideals of $R$ and $J(R)=\mathfrak{m}_{1} \cap \ldots \cap \mathfrak{m}_{k}$. Consider the following chain

$$
J(R) \supseteq J(R)^{2} \supseteq \ldots \supseteq J(R)^{n} \supseteq \ldots
$$

Suppose that $J(R)^{i} \supsetneqq J(R)^{i+1}$ for each $i \geq 1$. Then $|\mathbb{I}(J(R))|=\infty$. Since every ideal of $R$ is a vertex of $\mathbb{A G}(R)$, we conclude that $I_{1}=\operatorname{Ann}\left(\mathfrak{m}_{1}\right) \neq(0)$ and $I_{2}=\operatorname{Ann}\left(\mathfrak{m}_{2}\right) \neq(0)$. Also, $I_{1} \nsubseteq I_{2}$ and $I_{2} \nsubseteq I_{1}$ (otherwise, either $(0)=I_{1}\left(\mathfrak{m}_{1}+\mathfrak{m}_{2}\right)=I_{1} R$ or $(0)=$ $I_{2}\left(\mathfrak{m}_{1}+\mathfrak{m}_{2}\right)=I_{2} R$, a contradiction). Clearly $I_{1} J_{R}=I_{2} J(R)=\left(I_{1}+I_{2}\right) J(R)=(0)$ and so $K_{3,\left|\mathbb{I}(J(R))^{*}\right|}$ is a subgraph of $\mathbb{A} \mathbb{G}(R)$. Since $|\mathbb{I}(J(R))|=\infty, \gamma(\mathbb{A} \mathbb{G}(R))=\infty$, a contradiction. Therefore $J(R)^{n}=J(R)^{n+1}$ for some $n \geq 1$. Thus by Nakayama's lemma, $J(R)^{n}=(0)$. Suppose that $P$ is a prime ideal of $R$, since $J(R)^{n}=(0) \subseteq P$, there exists $\mathfrak{m}_{i}, 1 \leq i \leq n$ such that $\mathfrak{m}_{i}=P$. Thus every prime ideal of $R$ is a maximal ideal of $R$. Thus $R$ is an Artinian ring and by Theorem 2.7, $R$ has finitely many ideals. 
We conclude this paper with the following corollary.

Corollary 3.6 Let $R$ be a non-domain Noetherian local ring. If $\gamma(\mathbb{A} \mathbb{G}(R))<\infty$, then either $R$ is a Gorenstein ring or $R$ is an Artinian ring with finitely many ideals.

Proof. By Proposition 3.4 and Theorem 3.5 is clear.

\section{References}

[1] G. Aalipour, S. Akbari, M. Behboodi, R. Nikandish, M. J. Nikmehr and F. Shahsavari, The classification of the annihilating-ideal graph of a commutative ring, Algebra Collocuium, to appear.

[2] S. Akbari, A. Mohammadian, On the zero-divisor graph of a commutative ring, $J$. Algebra 274 (2004) 847-855.

[3] D. F. Anderson, P. S. Livingston, The zero-divisor graph of a commutative ring, $J$. Algebra 217 (1999) 434-447.

[4] D. D. Anderson, M. Naseer, Beck's coloring of a commutative ring, J. Algebra 159 (1993) 500-514.

[5] I. Beck, Coloring of commutative rings, J. Algebra 116 (1988) 208-226.

[6] M. Behboodi, Z. Rakeei, The annihilating-ideal graph of commutative rings I, $J$. Algebra Appl. to appear.

[7] M. Behboodi, Z. Rakeei, The annihilating-ideal graph of commutative rings II, $J$. Algebra Appl. to appear.

[8] P. R. Halmos. Linear Algebra problem Book, The mathematical Association of America, 1995.

[9] I. Kaplanski, Commutative Rings. rev. ed. Chicago: Univ. of Chicago Press, 1974.

[10] G. Ringel, Der vollstandinge paare graph auf nichtorientierbarenFlachen, J. Reine Angew. Math. 220 (1975) 89-93.

[11] G. Ringel, Map Color Theorem, Springer-verlag, New York, 1974.

[12] G. Ringel, J. W. T. Youngs, Solution of the heawood map-coloring problem,Proc. Nat. Acad. Sei. USA, 60 (1968) 438445.

[13] Hsin-Ju Wang, Zero-divisor graphs of genus one, J. Algebra 304 (2006) 666-678.

[14] Cameron Wickham, Classification of Rings with Genus One Zero-Divisor Graphs, Comm. Algebra 36 (2008) 325-345.

[15] Cameron Wickham, Rings whose zero-divisor graphs have positive genus, J. Algebra 321 (2009) 377-383.

[16] A. T. White, Graphs, Groups and Surfaces, North-Holland: Amsterdam, 1973. 